\title{
Influence of electrical steel sheet texirures on their magnetization curves
}

\author{
Witold Mazgaj, Adam Warzecha \\ Institute of Electromechanical Energy Conversion \\ Cracow University of Technology \\ Warszawska 24, 31-155 Kraków, Poland \\ e-mail: \{pemazgaj/pewarzec\}@cyfronet.pl
}

(Received: 26.06.2012, revised: 02.12.2012)

\begin{abstract}
The Goss texture is a characteristic feature of grain-oriented transformer steel sheets. Generator sheets, which are produced as non-oriented steel sheets, should have isotropic features. However, measurement results of generator sheets, confirmed by crystallographic studies, indicate that these sheets are characterized by certain, quite significant anisotropy. The first purpose of this paper is to present the influence of textures of generator and transformer steel sheets on their magnetization characteristics. The second aim is to propose a method which takes into account the sheet textures in the calculations of magnetization curves. In calculations of magnetization processes in electrical steel sheets, models in which the plane of a sheet sample is divided into an assumed number of specified directions are used. To each direction a certain hysteresis loop, the so-called direction hysteresis, is assigned. The parameters of these direction hystereses depend, among other things, on the texture type in these steel sheets. This paper discusses the method which calculates the parameters of these direction hystereses taking into account the given sheet texture. The proposed method gives a possibility of determining the magnetization characteristics for any direction of the field intensity changes.
\end{abstract}

Key words: electrical steel sheets, hysteresis loop, magnetization characteristics, Goss texture, anisotropy

\section{Introduction}

Almost all electrical steel sheets can be divided into two basic groups: generator sheets and transformer sheets. Generator sheets are used not only in the magnetic circuits of rotating machines but quite often they are used in the cores of small power transformers. Therefore, these sheets should have isotropic features in any direction on the sheet plane. However, special magnetic measurements carried out by means of both the RSST devices and the

* This is extended version of a paper which was presented at the 22th Symposium on Electromagnetic Phenomena in Nonlinear Circuits, Pula, Croatia, 26.06-29.06 2012. 
Epstein frame showed that generator steel sheets have certain anisotropic features. Crystallographic research, performed for several typical generator sheets with the use of the X-ray diffractometer, shows that an amount of sheet grains has a certain texture. The volume part of this texture is in the range from a few to a dozen or so percent of the volume of the whole sample. Various technological procedures in the manufacturing process of generator sheets are designed to achieve texture-free materials, but these sheets have not been produced so far on an industrial scale. The well-known Goss texture is a characteristic of transformer steel sheets which are made as grain-oriented sheets. This texture allows us to obtain high values of the flux density in the rolling direction. Obtaining of this texture in transformer sheets is an intended action in the manufacturing process. In some places of transformer cores the flux density is not parallel to the rolling direction (for example, in corners of cores). Then it causes the magnetization curves to differ from the curve measured for the rolling direction. The reason for these differences is the fact that the cubic-shape iron crystals have three axes of easy magnetization, so magnetic features depend also on the field intensity direction $[2,3]$.

Electrical steel sheets can be magnetized in different directions. Generally, this refers to generator sheets, although in transformer sheets the magnetic flux can change its direction, which takes place in a current overload. Thus, in many cases it is necessary to calculate the magnetic field distribution for different directions of the magnetic flux. This is possible using the appropriate model of the magnetization process which allows us to take into account the anisotropy properties of electrical steel sheets. In the papers $[6,7]$ the method of the magnetization modelling of soft magnetic materials was presented. This model can be applied to both rotational and alternating magnetization. In the proposed model the plane of a sample of an anisotropic sheet is divided into an assumed number of specified directions. To each direction a certain hysteresis loop, the so-called direction hysteresis, is assigned. These direction hystereses are described by such parameters as: saturation flux density, residual flux density, and coercive force. It is necessary to underline that these parameters and direction hystereses can not be measured and they differ from hysteresis of the electrical sheet sample. The parameters of the direction hystereses are calculated on the basis of measurements of the limiting and some partial hysteresis loops. It is also necessary to take into account the distribution function of the grains in the sample of a given anisotropic material. The above-mentioned distribution function is directly related to the texture which quite often occurs in generator sheets.

\section{Textures in electrical steel sheets}

In the Goss texture, the majority of iron grains (about 95-97\%) are situated with respect to the sheet plane as it is presented in Figure 1. In crystallography this texture is described as $\{110\}<100>$; this symbol shows which crystallographic planes and directions of iron crystals are parallel to the sheet plane and the rolling direction respectively [5]. Iron crystals have a cubic shape and each crystal has three axes of easy magnetization, which are described by the edges of the cube. One direction of these three axes is parallel to the rolling direction (RD), the other two axes are inclined to the sheet plane at an angle of $45^{\circ}$. The characteristic 
size of the Goss texture is the angle of $54.7^{\circ}$, which refers to the direction of the difficult magnetization. If the angle between the field intensity vector and the easy magnetization direction of the sheet plane (axis [100]) is less than $54.7^{\circ}$, the magnetic flux increases along this direction of easy magnetization $[4,10]$. In cases when transformer sheets are magnetized at angles greater than $54.7^{\circ}$ with respect to the rolling direction, the flux density increases mainly along the [010] and [001] axes. The purpose of procedures in the manufacturing process of transformer sheets is to strive for such an orientation of grains so that all grains form the Goss texture. In practice, a small percentage of the amount of grains is arranged in such a way that none of the easy magnetization axes is parallel to the rolling direction.

Fig. 1. Typical arrangement of iron crystals in sheets with the Goss texture

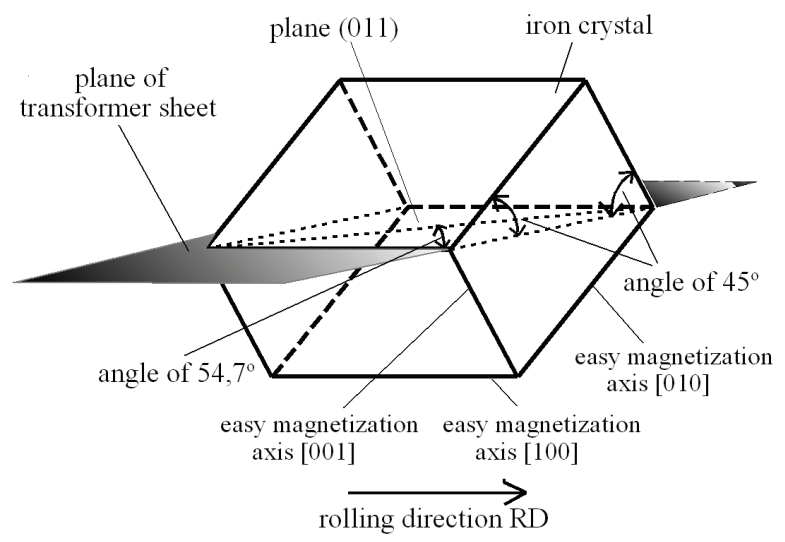

Generator sheets are produced as non-oriented sheets, but quite often they have certain anisotropic features. Texture studies were carried out for the chosen typical generator sheets with the thickness of $0.5 \mathrm{~mm}$ by means of the X-ray diffractometer ${ }^{1}$. During these tests, the stereographic projections are carried out and the reflections from the $\{100\}$ or $\{110\}$ plane of the given generator sheet sample are counted. Points with the same intensity of reflections are connected by lines, and it enables us to obtain the so-called pole figures which make possible the description of the texture type in generator sheets. The intensity of reflections in pole figures gives the possibility of estimation the volume of grains of a privileged crystallographic orientation. Figure 2 presents the $\{100\}$ type of pole figures of the M400-50A generator sheets (made in Sweden), and Figure 3 shows the pole figures of the M400-50A sheet (made in Russia). In both cases, tests were performed separately for the surface layer and the inner layer of these selected sheets; the thickness of the layers was about $0.15 \mathrm{~mm}$. The surface layer refers to the surface of the sheet distinct from the inner layer which is situated inside of the sheet. Both layers were obtained by appropriate mechanical - chemical treatment.

The pole figures presented in Figure 2 show that a certain amount of grains in the surface layer have the texture $\{011\}<100>$. It means that these grains are situated similarly as in the

\footnotetext{
${ }^{1}$ Texture studies were made in the Institute of Non Ferrous Metals in Gliwice, Light Metal Division in Skawina (Poland).
} 
Goss texture. Special software for the analysis of pole figures, used in crystallography, enable us to estimate the volumetric amount of this texture for about $8 \%$ of the whole volume of the sheet sample. A small portion of grains have the weak texture $\{125\}<210>$. In this case, none of the characteristic iron crystal planes is parallel to the sheet plane, and none of the easy magnetization axes is parallel to the rolling direction. One can say that the arrangement of these grains is rather similar to the so-called cubic texture [1]. In this texture two walls of cubic iron crystals are parallel to the sheet plane, and then the given generator sheet has the same magnetic properties in two perpendicular directions. In the inner layer of the M400-50A (Sweden) sheet the $\{001\}<210>$ crystallographic orientation is privileged and its volume part is estimated at around $6 \%$. It means that these grains have the cubic texture but the angle between one of the easy magnetization axes and the rolling direction is about $27^{\circ}$. Other grains, both in the surface and inner layers, are arranged randomly.

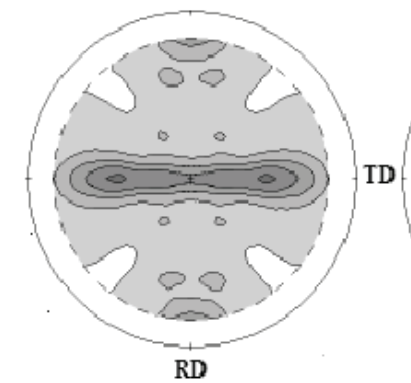

surface layer, Imax $=1.5$

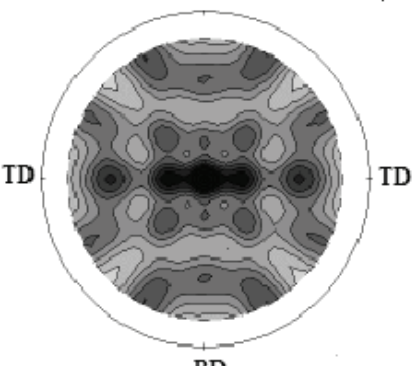

$\mathrm{RD}$

inner layer, $\operatorname{Imax}=6.4$

Fig. 2. Pole figures $\{100\}$ of the M400-50A sheet (made in Sweden); Imax denotes the maximum value of reflection intensity; intensity scale is linear, $\mathrm{RD}$ - rolling direction, $\mathrm{TD}$ - transverse direction

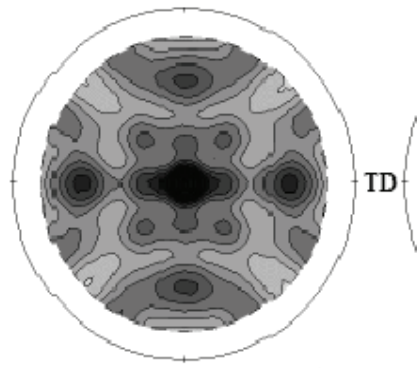

$\mathrm{RD}$

surface layer, $\quad \operatorname{Imax}=7.7$

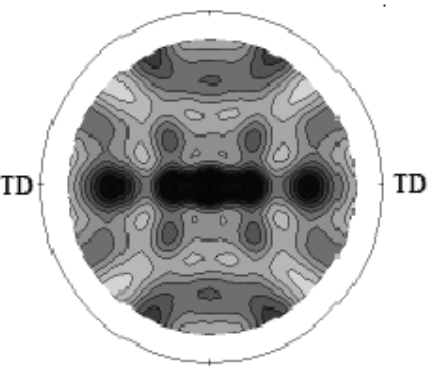

RD

inner layer, $\operatorname{Imax}=18.0$

Fig. 3. Pole figures $\{100\}$ of the M400-50A sheet (made in Russia); Imax denotes the maximum value of reflection intensity; intensity scale is linear, $\mathrm{RD}$ - rolling direction, $\mathrm{TD}$ - transverse direction

Pole figures of the M400-50A sheet produced in Russia (Fig. 3) show that in both surface and inner layers the crystallographic orientation $\{001\}<210>$ is privileged. The estimated 
volumetric part of this texture in the surface layer is about $9 \%$, and in the inner layer is about $19 \%$. In this sheet the weak texture $\{125\}<2 \overline{1} 0>$ also occurs.

Tests performed on samples of other generator sheets, e.g. M600-50A (Romania), M800-50A (Sweden), showed that the textures $\{001\}<210>$ and $\{125\}<2 \overline{1} 0>$ occur quite frequently. In some sheets the weak texture $\{111\}<2 \overline{1} 1>$ can occur. This orientation means that the planes $\{111\}$ are parallel to the rolling plane, and these planes are determined by three points of a cubic-shaped iron crystal which do not belong to the same crystal wall. It is necessary to underline that the volumetric amount of the privileged crystallographic orientation does not exceed a dozen or so percent of the total volume of the sheet sample.

\section{Taking into consideration the generator sheet textures}

It is known that the magnetization characteristics of the generator sheets with anisotropic properties depend on the direction of magnetization and on the sheet texture. The proposal of taking into account the anisotropy of grains was presented in the paper [7]. In that paper, the plane of an anisotropic sheet sample was divided into $n_{k}$ specified directions. Due to the sheet anisotropy, a different amount of iron grains was assigned to each specified direction. Although in generator sheets several privileged crystallographic orientations may exist, but the vast majority of grains are arranged randomly. Examples of grain distributions are presented in Figure 4.

Fig. 4. Examples of grain

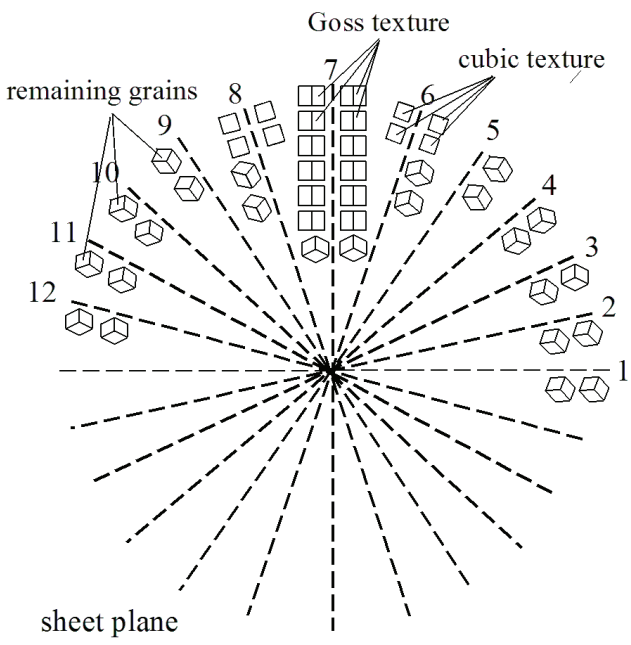

To each specified direction on the sheet plane the so-called direction hysteresis is assigned, which differs from the hysteresis of the whole anisotropic sheet sample. Therefore, the parameters of the direction hystereses like the saturation flux density $b_{s}$, the residual flux density $b_{r}$ 
and the coercive force $h_{c}$ must be determined. These parameters depend on the grain distribution function in the anisotropic sheet sample.

If the given generator sheet has a few textures then for each orientation the individual grain distribution function $d(k)$ should be determined in the following form (assuming 12 individual directions)

$$
d(k)=d_{1}, d_{2}, \ldots, d_{k}, \ldots, d_{11}, d_{12},
$$

where $d_{k}$ denotes the relative amount of grains which are assigned to the $k$-th direction.

It is worth emphasizing that most of the $d$ values are equal to zero because the grains of the given textures are not associated with all directions. As previously mentioned, the vast majority of grains are arranged randomly. For this part of grains the distribution function has the same value for all individual directions, and this value equals

$$
d_{r}=\left(1-V_{1}-V_{2}\right) / 12,
$$

where $V_{1}, V_{2}$ denote the relative volume part (with respect to the whole volume of the given sheet sample) of the two given textures.

If we assume that in the given sheet two textures occur, and $d_{1 k}, d_{2 k}$ denote the value of the grain distribution function for these two textures, then the condition

$$
\sum_{k=1}^{12}\left(d_{1 k}+d_{2 k}\right)+12 d_{r}=1
$$

must be fulfilled.

The flux densities of the direction hystereses reach the saturation state $b_{s}$ if the field intensity increases along one of individual direction. In this case the flux density vectors are situated along the magnetic field intensity vector, and the resultant flux density $B_{s}$ of the sheet sample is the algebraic sum of the saturation flux densities of all direction hystereses. Then the saturation flux density $b_{s k}$ for the given $k$-th direction equals

$$
b_{s k}=\left(d_{1 k}+d_{2 k}+d_{r}\right) B_{s},
$$

where $B_{s}$ is the saturation flux density, determined by measurements, of the anisotropic generator sheet.

If the field intensity is decreased from the saturation state to zero, the flux density vectors of the direction hystereses lie along individual directions, and the length of these vectors are equal to the residual flux density $b_{r k}$ of the direction hystereses. The residual flux densities of the direction hystereses have different values, and they depend on the grain distribution function. The resultant residual flux density of the whole sheet sample is the vector sum of the residual flux densities of the individual directions, and the following relation can be written as follows (assuming that the field intensity varies along the direction of number 7 in Fig. 4)

$$
B_{r}=b_{r 2} \cos 5 \alpha+b_{r 3} \cos 4 \alpha+\ldots+b_{r 7}+\ldots+b_{r 11} \cos 4 \alpha+b_{r 12} \cos 5 \alpha,
$$

where $B_{r}$ is the residual flux density of the anisotropic sheet (determined on the basis of 
measurements), $b_{r k}$ denotes the residual flux density in $k$-th direction, and $\alpha$ is an angle between two neighbouring directions.

Unlike the case in which the texture is not distinguished, the residual flux densities $b_{r k}$ in the individual directions are algebraic sums of the residual flux densities relating to different textures and of the residual flux density of the group of randomly arranged grains and

$$
\begin{aligned}
& b_{r 2}=b_{r 12}+b_{r 22}+a b_{r r}, \\
& b_{r 3}=b_{r 13}+b_{r 23}+a b_{r r}, \\
& b_{r 7}=b_{r 17}+b_{r 27}+a b_{r r}, \\
& b_{r 12}=b_{r 112}+b_{r 212}+a b_{r r},
\end{aligned}
$$

where $b_{r 1 k}, b_{r 2 k}$ denote the residual flux densities in $k$-th direction for the first and second texture respectively, $b_{r r}$ represents the residual flux density in each direction relating to the grains which are situated randomly, and $a=\cos 27^{\circ}$.

The $b_{r 1}$ flux density does not change its value because it is assumed that the field intensity varies along the direction 7 which is perpendicular to the direction of number 1 . The existence of the coefficient $a$ of the residual flux densities $b_{r r}$ in the last relationships requires some explanation. One of the easy magnetization axes (cube edges) of iron grains which are distributed randomly is always inclined to the sheet plane at an angle no greater than the $45^{\circ}$. The resultant flux density vector of these grains is not parallel to the sheet plane. It can be proved that this vector is inclined to the sheet plane at an angle of about $27^{\circ}$.

For the first texture the residual flux density $b_{r 1 k}$ for the $k$-th direction can be written as

$$
b_{r 1 k}=b_{r j} N_{1 k}=b_{r j} N \frac{N_{1 k}}{N}=b_{r j} N d_{1 k}=b_{r z} d_{1 k},
$$

where $b_{r j}$ is the residual flux density of a grain, $N_{1 k}$ determines the amount of grains of the first texture which are oriented in the $k$-th direction, $N$ denotes the amount of all grains in the anisotropy sheet sample, $d_{1 k}$ is the value of the grain distribution function of the first texture, and $b_{r z}$ is a certain auxiliary residual flux density.

Similar relationship as (7) can be written for the second texture and for the randomly distributed grains. In the latter case, the grain distribution function has the same value for each direction. Taking into account the last relationship, the Equation (5) can be written in the following form

$$
\begin{gathered}
B_{r}=b_{r z}\left[\left(d_{12}+d_{22}+a d_{r}\right) \cos 5 \alpha+\left(d_{13}+d_{23}+a d_{r}\right) \cos 4 \alpha+\ldots\right. \\
\left.+\left(d_{17}+d_{27}+a d_{r}\right)+\ldots+\left(d_{111}+d_{211}+a d_{r}\right) \cos 4 \alpha+\left(d_{112}+d_{212}+a d_{r}\right) \cos 5 \alpha\right] .
\end{gathered}
$$

Hence, the auxiliary residual flux density $b_{r z}$ equals 


$$
\begin{gathered}
b_{r z}=B_{r} /\left[\left(d_{12}+d_{22}+a d_{r}\right) \cos 5 \alpha+\left(d_{13}+d_{23}+a d_{r}\right) \cos 4 \alpha+\ldots\right. \\
\left.+\left(d_{17}+d_{27}+a d_{7}\right)+\ldots+\left(d_{111}+d_{211}+a d_{r}\right) \cos 4 \alpha+\left(d_{112}+d_{212}+a d_{r}\right) \cos 5 \alpha\right] .
\end{gathered}
$$

As mentioned previously, the residual flux densities $b_{r k}$ in the individual directions are algebraic sums of the residual flux densities relating to different textures and of the residual flux density of the group of randomly arranged grains. Thus on the basis of relation (7) it may be stated that the residual flux densities of the direction hystereses are equal to the expression $b_{r z}\left(d_{1 k}+d_{2 k}+a d_{r}\right)$. It is necessary to underline that the residual flux densities of the direction hystereses are different from the residual flux density determined on the basis of measurements of the whole sheet sample. In this case, they are the projections of the vector $\overrightarrow{\mathbf{B}}_{\mathbf{r}}$ on individual directions.

The coercive force is a certain field intensity value needed to move the domain walls through potential barriers until the resultant flux density of the total sheet sample is equal to zero [9]. The coercive forces of generator and also transformer steel sheets are relatively low. Therefore, rotations of flux density vectors towards to the direction of the field intensity can be neglected. In order to calculate the coercive force $h_{c}$ of the direction hystereses it is assumed that the curves of direction hysteresis loops are linear in a certain range. The coercive forces $h_{c}$ of the direction hystereses do not depend directly on the distribution function of iron grains in the generator sheets, and they can be determined on the basis of the relationship derived in $[6,7]$

$$
h_{c}=H_{c} \frac{b_{r 2} \cos ^{2} 5 \alpha+b_{r 3} \cos ^{2} 4 \alpha+\ldots+b_{r 7}+\ldots+b_{r 11} \cos ^{2} 4 \alpha+b_{r 12} \cos ^{2} 5 \alpha}{b_{r 2} \cos 5 \alpha+b_{r 3} \cos 4 \alpha+\ldots+b_{r 7}+\ldots+b_{r 11} \cos 4 \alpha+b_{r 12} \cos 5 \alpha}
$$

where $H_{c}$ denotes the coercive force of an generator sheet determined by measurement along the rolling direction, $b_{r k}$ is the residual flux density of the direction hysteresis in $k$-th direction. Calculations of direction hysteresis parameters with taking into account privileged crystallographic orientations allow us to determine magnetization curves for any direction. It is worth underlining that the resultant flux density in the sheet sample is the vector sum of the flux densities of the specified directions [8]. The parameters of direction hystereses were determined for the generator sheet M400-50A manufactured in Sweden $\left(B_{s}=1.8 \mathrm{~T}\right.$ at $10000 \mathrm{~A} / \mathrm{m}$, $B_{r}=1.05 \mathrm{~T}$, and $\left.H_{c}=60 \mathrm{~A} / \mathrm{m}\right)$ and for the sheet M400-50A produced in Russia $\left(B_{s}=1.8 \mathrm{~T}\right.$ at $\left.10000 \mathrm{~A} / \mathrm{m}, B_{r}=0.85 \mathrm{~T}, H_{c}=85 \mathrm{~A} / \mathrm{m}\right)$. The hysteresis loops for the rolling direction and transverse direction for both sheets were determined with the use of the rotational magnetization model $[6,7]$ with taking into account the sheet textures. These hysteresis loops were compared with measured loops ${ }^{2}$ (Fig. 5, 6).

\footnotetext{
${ }^{2}$ Hysteresis loops were measured in Laboratory of Magnetic Measurements in Bochnia (Poland)
} 

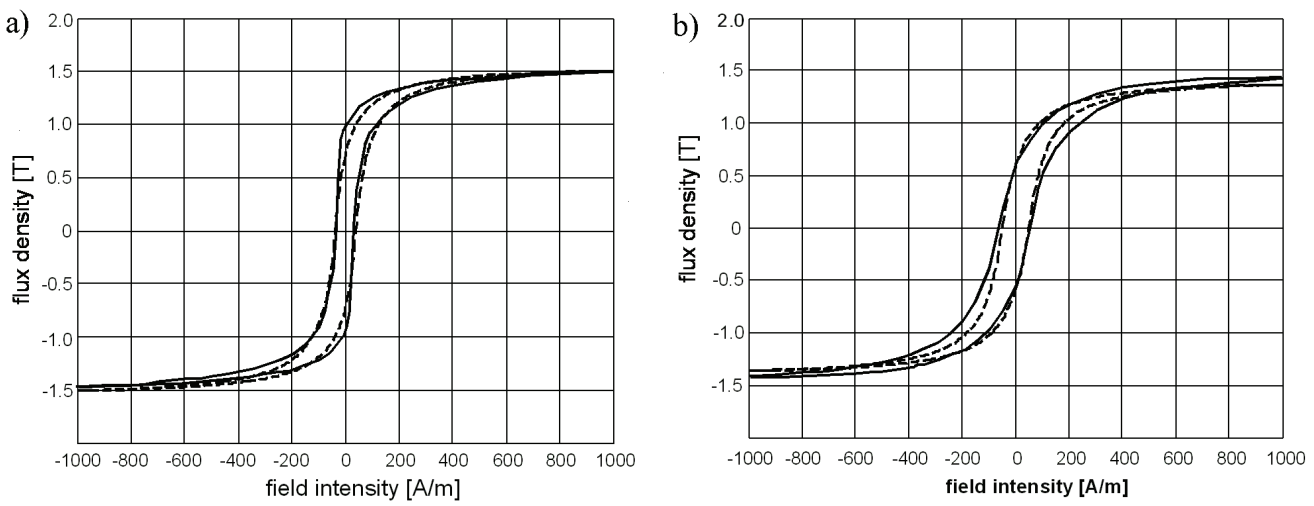

Fig. 5. Hysteresis loops of the generator sheet M400-50A (Sweden): a) for the rolling direction, b) for the transverse direction; continuous lines - loops determined by measurement, dashed lines - calculated loops
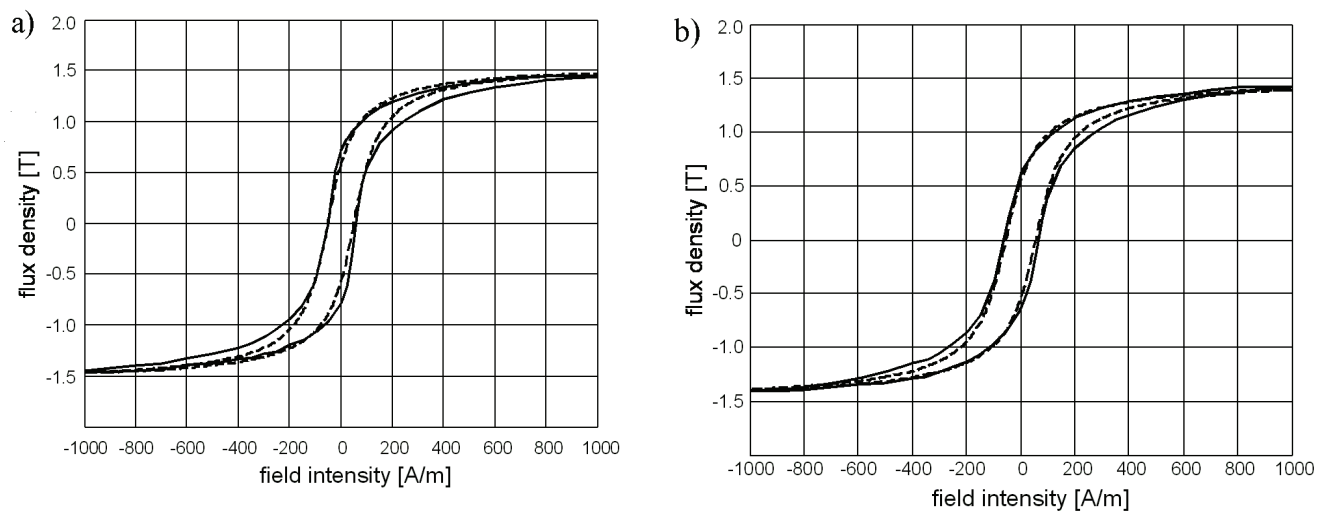

Fig. 6. Hysteresis loops of the generator sheet M400-50A (Russia): a) for the rolling direction, b) for the transverse direction; continuous lines - loops determined by measurement, dashed lines - calculated loops

\section{Calculation of magnetization curves in steel sheets with the Goss texture}

Taking into account the impact of the Goss texture in transformer steel sheets on magnetization characteristics is simpler than in the case of generator sheets. In practice, approximately 95-97\% of grains in transformer sheets have the Goss texture. It means that one of the easy magnetization axes of iron grains is parallel to the sheet plane and to the rolling direction. Without making a serious error it can be assumed that the remaining part of grains is distributed uniformly with respect to other directions. Thus, the value of the distribution function for the rolling direction ranges from 0.95 to 0.97 and from 0.0027 to 0.0045 for other directions. 
In cubic-shaped iron crystals, the magnetization process occurs along this magnetization axis which is closest to the field intensity direction. Figure 7 shows, for example, the flux density vectors in specified directions on the transformer sheet plane when the field intensity vector $H$ is parallel to the rolling direction $\mathrm{RD}$. In this assumed case the angles between directions $1,2,3$ and directions 11,12 are bigger than $54.7^{\circ}$, so the magnetization process in grains assigned to the above-mentioned directions occurs along the axes [010] and [001]. The field intensities for individual directions can be written as follows:

$$
\begin{gathered}
h_{1}=H \cos 45^{\circ}, \\
h_{2,12}=H \cos 45^{\circ} \cos \alpha, \\
h_{3,11}=H \cos 45^{\circ} \cos 2 \alpha, \\
h_{4,10}=H \cos 3 \alpha, \\
h_{7}=H,
\end{gathered}
$$

where indexes at $h$ are the numbers of individual directions, and $\alpha$ denotes, as before, the angle between two neighbouring directions.

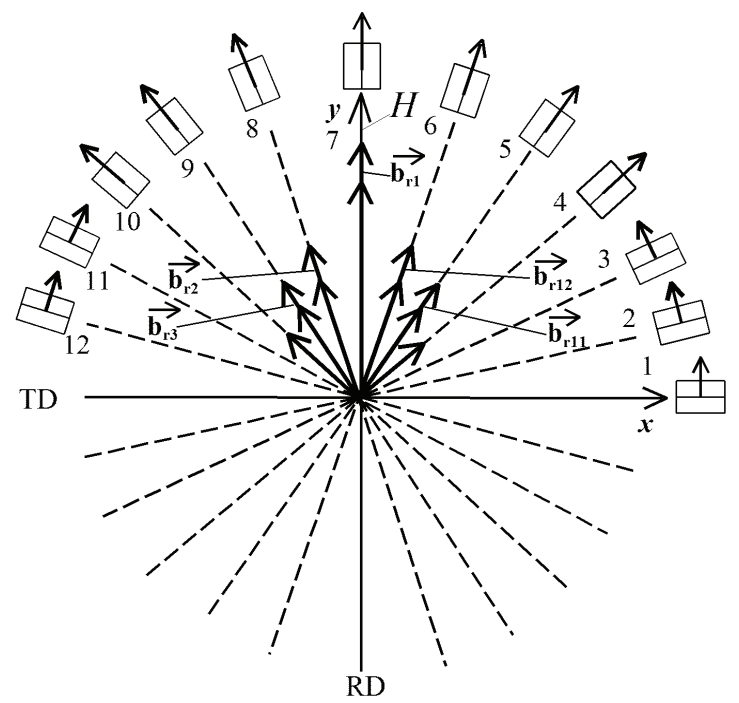

Fig. 7. Flux density vectors in a sheet sample with the Goss texture when the field intensity vector is parallel to the rolling direction $\mathrm{RD}$

The residual flux density of the given sheet sample with the Goss texture is the vector sum of the residual flux densities in all specified directions, and it can be written in the following form (assuming that the field intensity changes along the direction 7) 


$$
\begin{aligned}
B_{r}= & b_{r 1} \cos 45^{\circ}+2\left(b_{r 2,12} \cos 45^{\circ} \cos \alpha+b_{r 3,11} \cos 45^{\circ} \cos 2 \alpha+\ldots\right. \\
& \left.+b_{r 4,10} \cos 3 \alpha+b_{r 5,9} \cos 2 \alpha+b_{r 6,8} \cos \alpha\right)+b_{7},
\end{aligned}
$$

where $B_{r}$ is the residual flux density determined by measurement of the whole transformer sheet for the rolling direction, $b_{r k}$ denotes the residual flux density in the $k$-th direction.

The flux density vectors of the directions $1,2,3,11$ and 12 form the angles $45^{\circ}$ with respect to the sheet plane. Thus, determining the resultant flux density of the whole sheet sample with Goss texture, the length of these vectors must be multiplied by $\cos 45^{\circ}$, additionally for the directions 2 and 12 by $\cos \alpha$, and for directions 3 and 11 by $\cos 2 \alpha$.

Proceeding similarly as for the generator sheets the auxiliary residual flux density $b_{r z}$ can be expressed as

$$
\begin{aligned}
b_{r z} & =B_{r} /\left[d_{r} \cos 45^{\circ}+2\left(d_{r} \cos 45^{\circ} \cos \alpha+d_{r} \cos 45^{\circ} \cos 2 \alpha+\ldots\right.\right. \\
& \left.\left.+d_{r} \cos 3 \alpha+d_{r} \cos 2 \alpha+d_{r} \cos \alpha\right)+d_{R D}\right]
\end{aligned}
$$

where $d_{R D}$ is the value of the distribution function of grains for the rolling direction $\mathrm{RD}, d_{r}$ denotes the value of the distribution function for remaining directions.

After calculating the $b_{r z}$ value on the basis of the last equation, the residual flux densities of direction hystereses can be determined by multiplying the value $b_{r z}$ by $d_{R D}$ or by $d_{r}$ respectively.

The vector sum of flux densities of all direction hystereses is equal to zero if the field intensity in the whole sheet sample is equal to the coercive force $H_{c}$. This condition can be written as

$$
\begin{aligned}
& b_{1} \cos 45^{\circ}+2\left(b_{1,22} \cos 45^{\circ} \cos \alpha+b_{3,11} \cos 45^{\circ} \cos 2 \alpha+\ldots\right. \\
& \left.+b_{4,10} \cos 3 \alpha+b_{5,9} \cos 2 \alpha+b_{6,8} \cos \alpha\right)+b_{7}=0 .
\end{aligned}
$$

As it was assumed previously, in a certain range the flux densities of the direction hystereses are linear functions with respect to the field intensity. Taking this into account and using the method described in [7], the coercive force $h_{c}$ of the direction hystereses is equal to

$$
h_{c}=H_{c} \frac{L}{M},
$$

where

$$
\begin{aligned}
L= & b_{r 1} \cos ^{2} 45^{\circ}+2\left(b_{r 2,12} \cos ^{2} 45^{\circ} \cos ^{2} \alpha+b_{r 3,11} \cos ^{2} 45^{\circ} \cos ^{2} 2 \alpha+\ldots\right. \\
& +b_{r 4,10} \cos ^{2} 3 \alpha+b_{r 5,9} \cos ^{2} 2 \alpha+b_{r 6,8} \cos ^{2} \alpha, \\
M & =b_{r 1} \cos 45^{\circ}+2\left(b_{r 2,12} \cos 45^{\circ} \cos \alpha+b_{r 3,11} \cos 45^{\circ} \cos 2 \alpha+\ldots\right. \\
& \left.+b_{r 4,10} \cos 3 \alpha+b_{r 5,9} \cos 2 \alpha+b_{r 6,8} \cos \alpha\right)+b_{r 7} .
\end{aligned}
$$


One of the typical transformer sheet with the Goss texture is the sheet M089-27N (produced in Poland) with the following magnetic parameters: saturation flux density $-2.0 \mathrm{~T}$, residual flux density and coercive force for the rolling direction $-1.42 \mathrm{~T}, 8 \mathrm{~A} / \mathrm{m}$ respectively. Assuming that the texture degree is about $95 \%$, the coercive force $h_{c}$ of direction hystereses equals $7.95 \mathrm{~A} / \mathrm{m}$, and the residual flux densities $b_{r}$ for the rolling and remaining directions are $1.38 \mathrm{~T}$ and $0.015 \mathrm{~T}$ respectively. Examples of the flux density changes in the given transformer sheet M089-27N, which was magnetized in three characteristic directions, are shown in the Figure 8. Continuous lines represent magnetization curves calculated with taking into account the features of the Goss texture, and dashed lines refer to the measured curves. Continuous lines represent magnetization curves calculated using the model of the rotational magnetization [7] with taking into account relations (11) - (15), and dashed lines refer to the measured curves.

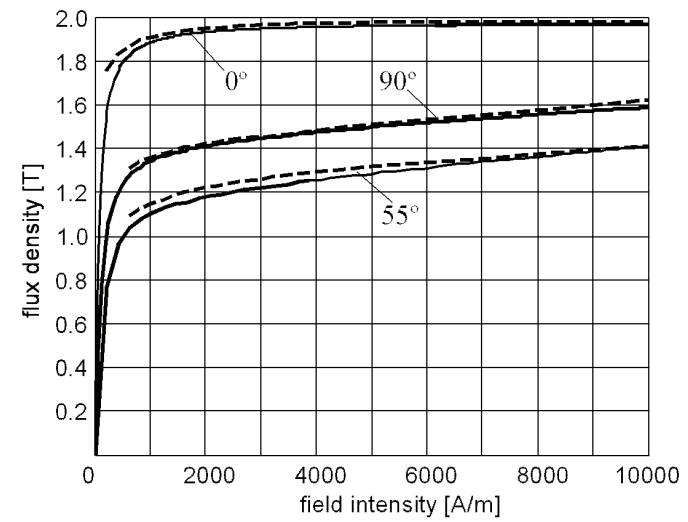

Fig. 8. Magnetization curves of the transformer sheet M089-27N; continuous lines - calculated curves, dashed lines - measured curves

\section{Conclusions}

Changes of the flux density in electrical steel sheets depend not only on the field intensity value but also on the texture types which can occur in these sheets. This is important if the direction of the field intensity is not parallel to the rolling direction, especially during the rotational magnetization.

The sheet texture is taken into account by an appropriate modification of the previously defined grain distribution function. Consequently, the parameters of the direction hystereses depend on the texture type. However, it should be noted that the error associated with the texture determination can equal to a dozen or so percent.

Calculations of magnetization processes become more complicated when the grains are not arranged in a manner typical of the Goss texture or cubic texture, which quite often occurs in some generator sheets. In this case, the so-called Euler's angles are helpful. These angles allow us to transform the co-ordinates of points from the co-ordinate system which is connected 
with the rolling and transverse directions to the system which is assigned to the three axes of cubic-shape iron crystals.

It is necessary to underline that for the comprehensive approach to the magnetic field distribution the eddy currents should be taken into account, because eddy currents influence magnetic field distribution in electric steel sheets.

\section{Acknowledgments}

This paper was supported by research grant number DEC-2011/01/B/ST7/04479 "Modelling of nonlinearity, hysteresis, and anisotropy of magnetic cores in electromechanical converters with rotating magnetic field" financed from National Science Centre, (Poland)

\section{References}

[1] Beckley P., Electrical Steels for Rotating Machines. Bell \& Bain Ltd., Glasgow (2002).

[2] Bozorth R.M., Ferromagnetism. IEEE Press, New York (1978).

[3] Brailsford F., Physical Principles of Magnetism. Butler \& Tanner Ltd., London (1966).

[4] Fiorillo F., Dupré L.R., Appino C., Rietto A.M., Comprehensive Model of Magnetization Curve, Hysteresis Loops, and Losses in Any Direction in Grain-Oriented Fe-Si. IEEE Transactions on Magnetics 38(3): 1467-1475 (2002).

[5] Kelly A., Groves G.W., Crystallography and crystal defects. Longman, London (1970).

[6] Mazgaj W., Modelling of the rotational magnetization process of soft ferromagnetic materials. Archives of Electrical Engineering LVII(3-4): 231-243 (2008).

[7] Mazgaj W., Modelling of rotational magnetization in anisotropic sheets. COMPEL: The International Journal for Computation and Mathematics in Electrical and Electronic Engineering 30(3): 957-967 (2011).

[8] Mayergoyz I.D., Mathematical Models of Hysteresis and Their Applications, Elsevier Inc., New York (2003).

[9] Morrish A.H., The Physical Principles of Magnetism. John Wiley and Sons, Inc., New York, NY (1965).

[10] Pfützner H., Rotational Magnetization and Rotational Losses of Grain Oriented Silicon Steel Sheets - Fundamental Aspects and Theory. IEEE Transactions on Magnetics 30(5): 2802-2807 (1994). 